\title{
Generalized Reissner-Nordström solution in Metric-Affine Gravity
}

\author{
Alfredo Macías ${ }^{\diamond *}$ and José Socorro ${ }^{\$ \dagger}$ \\ • Departamento de Física, \\ Universidad Autónoma Metropolitana-Iztapalapa, \\ Apartado Postal 55-534, 09340 México, D.F., MEXICO \\ * Instituto de Ciencias Nucleares, Universidad Nacional Autónoma de México \\ Apartado Postal 70-543, 04510 México D.F, MEXICO \\ \$ Instituto de Física de la Universidad de Guanajuato, \\ Apartado Postal E-143, C.P. 37150, León, Guanajuato, MEXICO
}

(September 14, 2021)

\begin{abstract}
We present the generalized Reissner-Nordström solution of the field equations of metric-affine gravity (MAG), endowed with electric and magnetic charges, as well as with gravito-electric and gravito-magnetic charges and a cosmological constant term. Moreover, the case $M=e_{o}$, i.e. mass equal to electric charge and $\lambda=0$, corresponds to an electrically and magnetically charged monopole. Also further multipole solutions are obtained. The charge assignments of the solutions is discussed. file mono8.tex 04.04.1999.
\end{abstract}

PACS no.: 04.50.+h; 04.20.Jb; 03.50.Kk

*E-mail: amac@xanum.uam.mx,macias@nuclecu.unam.mx

†E-mail: socorro@ifug4.ugto.mx 


\section{INTRODUCTION}

Examples in which spacetime might become non-Riemannian above Planck energies occur in string theory [1 5] or in the very early universe in the inflationary model [6 10]. The simplest such geometry is metric-affine geometry, in which nonmetricity appears as a field strength, side by side with curvature and torsion [11]. The Einstein-Cartan theory yields a non-Riemannian spacetime already for $10^{52} \mathrm{~g} / \mathrm{cm}^{3}$, well below the Planck density of about $10^{92} \mathrm{~g} / \mathrm{cm}^{3}$ [12].

As it is well known [13], MAG represents a gauge theory of the 4-dimensional affine group enriched by the existence of a metric. As a gauge theory, it finds its appropriate form if expressed with respect to arbitrary frames or coframes. We have then the metric $g_{\alpha \beta}$, the coframe $\vartheta^{\alpha}$, and the connection 1 -form $\Gamma_{\alpha}{ }^{\beta}$ (with values in the Lie algebra of the 4 dimensional linear group $G L(4, R))$ as independent field variables. Spacetime is described by a metric-affine geometry with the gravitational field strengths nonmetricity $Q_{\alpha \beta}:=-D g_{\alpha \beta}$, torsion $T^{\alpha}:=D \vartheta^{\alpha}$, and curvature $R_{\alpha}^{\beta}:=d \Gamma_{\alpha}{ }^{\beta}-\Gamma_{\alpha}^{\gamma} \wedge \Gamma_{\gamma}{ }^{\beta}$.

On the other hand, we do not believe that at the present state of the universe the geometry of spacetime is described by a metric-affine one. We rather think, and there is good experimental evidence, that the present-day geometry is metric-compatible, i.e., its nonmetricity vanishes. In earlier epochs of the universe, however, when the energies of the cosmic "fluid" were much higher than today, we expect scale invariance to prevail and the canonical dilation (or scale) current of matter, the trace of the hypermomentum current $\Delta_{\gamma}^{\gamma}$, is coupled, according to MAG, to the Weyl covector $Q_{\gamma}^{\gamma}$. By the same token, shear type excitations of the material multispinors (Regge trajectory type of constructs) are expected to arise, thereby liberating the (metric-compatible) Riemann-Cartan spacetime from its constraint of vanishing nonmetricity $Q_{\alpha \beta}=0$. Tresguerres [14] has proposed a simple cosmological model of Friedmann type which carries a metric-affine geometry at the beginning of the universe, the nonmetricity of which dies out exponentially in time. That is the kind of thing we expect.

Moreover, for getting a deeper understanding of the meaning and the possible consequences of MAG, a search for exact solutions appears indispensable. The search for exact solutions of MAG is still in its infancy. It began with the work of Tresguerres [15,16], Tucker and Wang [17], Obukhov et al. [18], Vlashinsky et al. [19], and of Puntigam et al. [20]. Macías et al. [21], and Socorro et al. [22] mapped the Einstein-Maxwell sector of dilaton-gravity, emerging from low energy string theory, and found new soliton and multipole solutions of MAG. However, it is important to note that in order to incorporate the scalar dilaton field, one could, for instance, generalize the torsion kink of Baekler et al. 23], an exact solution with an external massless scalar field, or one could turn to the axi-dilatonic sector of MAG [24. García et al. 25] found the Plebański-Demiański class of solutions in MAG. Moreover, solutions implying the existence of torsion shock waves have already been found by García et al. 26]. For a review of all known exact solutions in MAG see Ref. [27].

It has been demonstrated that for each Einstein-Maxwell solution (metric plus electromagnetic potential 1-form), if the electric charge is replaced by the strong gravito-electric charge and if a suitable constraint on the coupling constants is postulated, an exact solution of MAG can be created. Even more so, the MAG model considered here can be reduced to an effective Einstein-Proca system [28,29. Now the meaning of the constraint between 
the different coupling constants becomes immediately clear: If we put $m^{2}=0$, then the Einstein-Proca system becomes an Einstein-Maxwell system. Moreover, it is quite useful to find electrically charged versions of a MAG solution [30,31] in order to illustrate the coupling of the electromagnetic field to the post-Riemannian structures of a metric-affine spacetime.

In this paper, we present a generalized Reissner-Nordström solution in MAG endowed with electric, magnetic, gravito-electric and gravito-magnetic charges and cosmological constant. Moreover, the electrically and magnetically charged gravito-electric and gravitomagnetic MAG monopole solutions contained on it are also discussed. It is worthwhile to stress the well know fact [32] that in the general relativistic context, the Reissner-Nordström solution is very important since it represents the gravitational field of a static charged mass. It is the only static solution which can be thought of as a static black hole endowed with mass and electric charge. Switching off the charge, it reduces to the Schwarzschild solution.

The plan of the paper is as follows: In Sec. II we review the MAG Lagrangian. In Sec. III we present the solitonic monopole field configuration in MAG. In Sec. IV electrically and magnetically charged strong gravito-magnetic monopole solutions are presented. In Sec. V we transform the monopole solution to Schwarzschild-like coordinates. In Sec. VI a generalized Reissner-Nordström solution in MAG is presented. In Sec. VII we display further multipole solutions. In Sec. VIII the charge assignment of the solutions is given. In Sec. IX the results are discussed.

\section{QUADRATIC MAG LAGRANGIAN}

Propagating post-Riemannian gauge interactions in MAG can be consistently constructed by adding terms quadratic in in $Q_{\alpha \beta}, T^{\alpha}, R_{\alpha}{ }^{\beta}$ to the Hilbert-Einstein type Lagrangian and the term with the cosmological constant.

In the first order formalism we are using, higher order terms in the gauge field strengths $Q_{\alpha \beta}, T^{\alpha}, R_{\alpha}^{\beta}$, i.e. cubic and quartic ones etc. would preserve the second order of the field equations. However, the quasilinearity of the gauge field equations would be destroyed and, in turn, the Cauchy problem would be expected to be ill-posed. Therefore we do not go beyond a gauge Lagrangian which is quadratic in the gauge field strengths. Moreover, a quadratic Lagrangian is already so messy that it would be hard to handle a still more complex one anyway.

The most general parity conserving quadratic Lagrangian which is expressed in terms of the $4+3+11$ irreducible pieces of $Q_{\alpha \beta}, T^{\alpha}, R_{\alpha}{ }^{\beta}$, respectively (see [13]):

$$
\begin{aligned}
V_{\mathrm{MAG}} & =\frac{1}{2 \kappa}\left[-a_{0} R^{\alpha \beta} \wedge \eta_{\alpha \beta}-2 \lambda \eta+T^{\alpha} \wedge^{*}\left(\sum_{I=1}^{3} a_{I}{ }^{(I)} T_{\alpha}\right)\right. \\
& +2\left(\sum_{I=2}^{4} c_{I}{ }^{(I)} Q_{\alpha \beta}\right) \wedge \vartheta^{\alpha} \wedge^{*} T^{\beta}+Q_{\alpha \beta} \wedge^{*}\left(\sum_{I=1}^{4} b_{I}{ }^{(I)} Q^{\alpha \beta}\right) \\
& \left.\left.+b_{5}{ }^{\left({ }^{(3)}\right.} Q_{\alpha \gamma} \wedge \vartheta^{\alpha}\right) \wedge^{*}\left({ }^{(4)} Q^{\beta \gamma} \wedge \vartheta_{\beta}\right)\right] \\
& -\frac{1}{2 \rho} R^{\alpha \beta} \wedge^{*}\left(\sum_{I=1}^{6} w_{I}{ }^{(I)} W_{\alpha \beta}+w_{7} \vartheta_{\alpha} \wedge\left(e_{\gamma}{ }^{(5)} W_{\beta}^{\gamma}\right)\right.
\end{aligned}
$$




$$
\left.\left.\left.+\sum_{I=1}^{5} z_{I}^{(I)} Z_{\alpha \beta}+z_{6} \vartheta_{\gamma} \wedge\left(e_{\alpha}\right\rfloor^{(2)} Z_{\beta}^{\gamma}\right)+\sum_{I=7}^{9} z_{I} \vartheta_{\alpha} \wedge\left(e_{\gamma}\right\rfloor^{(I-4)} Z_{\beta}^{\gamma}\right)\right) .
$$

The constant $\lambda$ is the cosmological constant, $\rho$ the strong gravity coupling constant, the constants $a_{0}, \ldots a_{3}, b_{1}, \ldots b_{5}, c_{2}, c_{3}, c_{4}, w_{1}, \ldots w_{7}, z_{1}, \ldots z_{9}$ are dimensionless. We have introduced in the curvature square term the irreducible pieces of the antisymmetric part $W_{\alpha \beta}:=R_{[\alpha \beta]}$ and the symmetric part $Z_{\alpha \beta}:=R_{(\alpha \beta)}$ of the curvature 2 -form. In $Z_{\alpha \beta}$, we have the purely post-Riemannian part of the curvature. Note the peculiar cross terms with $c_{I}$ and $b_{5}$.

The first and the second field equations of MAG read

$$
\begin{aligned}
D H_{\alpha}-E_{\alpha} & =\Sigma_{\alpha}, \\
D H^{\alpha}{ }_{\beta}-E^{\alpha}{ }_{\beta} & =\Delta^{\alpha}{ }_{\beta},
\end{aligned}
$$

where $\Sigma_{\alpha}$ and $\Delta^{\alpha}{ }_{\beta}$ are the canonical energy-momentum and hypermomentum current threeforms associated with matter. The left hand sides of (2.2)-(2.3) involve the two-forms of the gravitational gauge field momenta $H_{\alpha}$ and $H^{\alpha}{ }_{\beta}$ (gravitational "excitations"). We find them, together with $M^{\alpha \beta}$, by partial differentiation of the Lagrangian (2.1):

$$
\begin{aligned}
M^{\alpha \beta} & :=-2 \frac{\partial V_{\mathrm{MAG}}}{\partial Q_{\alpha \beta}}=-\frac{2}{\kappa}\left\{*\left(\sum_{I=1}^{4} b_{I}^{(I)} Q^{\alpha \beta}\right)+\frac{1}{2} b_{5}\left[\vartheta^{(\alpha} \wedge^{*}\left(Q \wedge \vartheta^{\beta)}\right)-\frac{1}{4} g^{\alpha \beta *}(3 Q+\Lambda)\right]\right. \\
& \left.+c_{2} \vartheta^{(\alpha} \wedge^{*(1)} T^{\beta)}+c_{3} \vartheta^{(\alpha} \wedge^{*(2)} T^{\beta)}+\frac{1}{4}\left(c_{3}-c_{4}\right) g^{\alpha \beta *} T\right\} \\
H_{\alpha} & :=-\frac{\partial V_{\mathrm{MAG}}}{\partial T^{\alpha}}=-\frac{1}{\kappa} *\left[\left(\sum_{I=1}^{3} a_{I}^{(I)} T_{\alpha}\right)+\left(\sum_{I=2}^{4} c_{I}{ }^{(I)} Q_{\alpha \beta} \wedge \vartheta^{\beta}\right)\right] \\
H_{\beta}^{\alpha} & :=-\frac{\partial V_{\mathrm{MAG}}}{\partial R_{\alpha}{ }^{\beta}}=\frac{a_{0}}{2 \kappa} \eta^{\alpha}{ }_{\beta}+\mathcal{W}^{\alpha}{ }_{\beta}+\mathcal{Z}^{\alpha}{ }_{\beta}
\end{aligned}
$$

where we introduced the abbreviations

$$
\mathcal{W}_{\alpha \beta}:=*\left(\sum_{I=1}^{6} w_{I}^{(I)} W_{\alpha \beta}\right), \quad \mathcal{Z}_{\alpha \beta}:=*\left(\sum_{I=1}^{5} z_{I}^{(I)} Z_{\alpha \beta}\right) .
$$

The three-forms $E_{\alpha}$ and $E^{\alpha}{ }_{\beta}$ describe the canonical energy-momentum and hypermomentum currents of the gauge fields themselves. They can be written as follows [13]:

$$
\begin{aligned}
E_{\alpha} & \left.\left.\left.\left.=e_{\alpha}\right\rfloor V_{\mathrm{MAG}}+\left(e_{\alpha}\right\rfloor T^{\beta}\right) \wedge H_{\beta}+\left(e_{\alpha}\right\rfloor R_{\beta}^{\gamma}\right) \wedge H_{\gamma}^{\beta}+\frac{1}{2}\left(e_{\alpha}\right\rfloor Q_{\beta \gamma}\right) M^{\beta \gamma} \\
E^{\alpha}{ }_{\beta} & =-\vartheta^{\alpha} \wedge H_{\beta}-M_{\beta}^{\alpha},
\end{aligned}
$$

where $\left.e_{\alpha}\right\rfloor$ denotes the interior product with a form.

\section{MONOPOLE FIELD CONFIGURATION IN MAG}

We search for exact solutions of the field equations belonging to the Lagrangian

$$
L=V_{\mathrm{MAG}}+V_{\mathrm{Max}}
$$


where $V_{\text {Max }}=-(1 / 2) F \wedge{ }^{\star} F$ is the Lagrangian of the Maxwell field and $F=d A$ is the electromagnetic field strength. Since the matter part $V_{\text {Max }}$ does not depend on the connection $\Gamma_{\alpha}{ }^{\beta}$, the hypermomentum $\Delta^{\alpha}{ }_{\beta}:=\delta V_{\text {Max }} / \delta \Gamma_{\alpha}{ }^{\beta}$ vanishes, i.e. $\Delta^{\alpha}{ }_{\beta}=0$, and the only external current is the electromagnetic energy-momentum current $\Sigma_{\alpha}$, given by

$$
\left.\left.\Sigma_{\alpha}=2 a_{0}\left(e_{\alpha}\right\rfloor V_{\mathrm{Max}}+\left(e_{\alpha}\right\rfloor F\right) \wedge H\right) .
$$

The monopole type solutions are found in terms of isotropic coordinates $(t, r, \theta, \phi)$, which lead to the coframe 21]

$$
\vartheta^{\hat{0}}=\frac{1}{f} d t, \quad \vartheta^{\hat{1}}=f d r, \quad \vartheta^{\hat{2}}=f r d \theta, \quad \vartheta^{\hat{3}}=f r \sin \theta d \varphi
$$

with one unknown function $f=f(r, \theta)$. The coframe is assumed to be orthonormal, that is, with the local Minkowski metric $o_{\alpha \beta}:=\operatorname{diag}(-1,1,1,1)=o^{\alpha \beta}$, yielding the spherically symmetric metric in isotropic form

$$
d s^{2}=o_{\alpha \beta} \vartheta^{\alpha} \otimes \vartheta^{\beta}=-\frac{1}{f^{2}} d t^{2}+f^{2}\left[d r^{2}+r^{2}\left(d \theta^{2}+\sin ^{2} \theta d \phi^{2}\right)\right] .
$$

As for the torsion and nonmetricity configurations, we concentrate on the simplest nontrivial case with shear. The nonmetricity, according to its irreducible decomposition, contains two covector pieces, namely ${ }^{(4)} Q_{\alpha \beta}=Q g_{\alpha \beta}$, the dilation piece, and

$$
\left.\left.{ }^{(3)} Q_{\alpha \beta}=\frac{4}{9}\left(\vartheta_{(\alpha} e_{\beta)}\right\rfloor \Lambda-\frac{1}{4} g_{\alpha \beta} \Lambda\right), \quad \text { with } \quad \Lambda:=\vartheta^{\alpha} e^{\beta}\right\rfloor \ell_{\alpha \beta},
$$

a proper shear piece [1] with $\ell_{\alpha \beta}:=Q_{\alpha \beta}-Q g_{\alpha \beta}$ the remaining traceless piece of the nonmetricity. Accordingly, our ansatz for the nonmetricity reads

$$
Q_{\alpha \beta}={ }^{(3)} Q_{\alpha \beta}+{ }^{(4)} Q_{\alpha \beta} .
$$

The torsion, in addition to its tensor piece, encompasses a vector and an axial vector piece. Let us choose only the vector piece as non-vanishing:

$$
\left.T^{\alpha}={ }^{(2)} T^{\alpha}=\frac{1}{3} \vartheta^{\alpha} \wedge T, \quad \text { with } \quad T:=e_{\alpha}\right\rfloor T^{\alpha} .
$$

Thus we are left with the three non-trivial one-forms $Q, \Lambda$, and $T$, which should not distinguish a direction in space in the spherically symmetric case. The following ansatz, for the above mentioned triplet of one-forms, turns out to be compatible with that condition,

$$
Q=k_{0}\left[N_{e} u(r, \theta) \vartheta^{\hat{0}}+N_{g} \frac{w(r, \theta)}{f r \sin \theta} \vartheta^{\hat{3}}\right]=\frac{k_{0}}{k_{1}} \Lambda=\frac{k_{0}}{k_{2}} T,
$$

with $N_{e}$ the gravito-electric charge and $N_{g}$ the gravito-magnetic one. The electromagnetic potential $A$ appropiate for this configuration can be expressed as follows [30,31]

$$
A=e_{o} u(r, \theta) \vartheta^{\hat{0}}+g_{o} \frac{w(r, \theta)}{f r \sin \theta} \vartheta^{\hat{3}},
$$

where $e_{o}$ is the electric charge and $g_{o}$ the magnetic charge. 


\section{ELECTRICALLY AND MAGNETICALLY CHARGED STRONG GRAVITO-MAGNETIC MONOPOLES}

In order to solve the equations arising from the MAG Lagrangian (2.1), we removed all other curvature square pieces by setting $w_{I}=0, z_{1}=z_{2}=z_{3}=z_{5}=0, b_{5}=0$ and $\lambda=0$ in the Lagrangian keeping the square of the segmental curvature in the Lagrangian 18,19.

We substitute the local metric $o_{\alpha \beta}$, the coframe (3.3), the nonmetricity and torsion (3.8) into the field equations (2.2), (2.3) of the Lagrangian (2.1). Provided the (rather weak) constraint

$$
\begin{aligned}
& 32 a_{0}^{2} b_{4}-4 a_{0} a_{2} b_{4}+64 a_{0} b_{3} b_{4}-32 a_{2} b_{3} b_{4}+48 a_{0} b_{4} c_{3}+24 b_{4} c_{3}^{2}+24 b_{3} c_{4}^{2} \\
& \quad+12 a_{0} a_{2} b_{3}+48 a_{0} b_{3} c_{4}-9 a_{0} c_{3}^{2}+18 a_{0} c_{3} c_{4}+3 a_{0} c_{4}^{2}+6 a_{0}^{2} a_{2}+24 a_{0}^{2} c_{4}=0,
\end{aligned}
$$

on the coupling constants is fulfilled, then we find an exact solution with

$$
f=1 \pm q u(r), \quad u(r)=1-\frac{1}{r}, \quad w=(1-\cos \theta) .
$$

Here $q$ is an arbitrary integration constant, and the coefficients $k_{0}, k_{1}, k_{2}$ in the ansatz (3.8) are determined by the dimensionless coupling constants of the Lagrangian:

$$
\begin{aligned}
& k_{0}=\left(\frac{a_{2}}{2}-a_{0}\right)\left(8 b_{3}+a_{0}\right)-3\left(c_{3}+a_{0}\right)^{2}, \\
& k_{1}=-9\left[a_{0}\left(\frac{a_{2}}{2}-a_{0}\right)+\left(c_{3}+a_{0}\right)\left(c_{4}+a_{0}\right)\right], \\
& k_{2}=\frac{3}{2}\left[3 a_{0}\left(c_{3}+a_{0}\right)+\left(8 b_{3}+a_{0}\right)\left(c_{4}+a_{0}\right)\right] .
\end{aligned}
$$

Then the constraint (4.1) can be put into the following more compact form

$$
b_{4}=\frac{a_{0} k+2 c_{4} k_{2}}{8 k_{0}}, \quad \text { with } \quad k:=3 k_{0}-k_{1}+2 k_{2},
$$

implying the following relation for $z_{4}$

$$
q^{2}=\kappa\left(e_{o}^{2}+g_{o}^{2}+z_{4} k_{0}^{2} \frac{N_{e}^{2}+N_{g}^{2}}{2 a_{0}}\right) .
$$

The factor $k$ is directly related to the antisymmetric piece of the Ricci 1 -form [18]. The electromagnetic potential can now be written as

$$
A=e_{o}\left(1-\frac{1}{r}\right) \vartheta^{\hat{0}}+g_{o} \frac{1-\cos \theta}{f r \sin \theta} \vartheta^{\hat{3}} .
$$

Collecting our results, the nonmetricity and the torsion read as follows:

$$
\begin{gathered}
\left.Q^{\alpha \beta}=\left[k_{0} o^{\alpha \beta}+\frac{4}{9} k_{1}\left(\vartheta^{(\alpha} e^{\beta)}\right\rfloor-\frac{1}{4} o^{\alpha \beta}\right)\right]\left[N_{e}\left(1-\frac{1}{r}\right) \vartheta^{\hat{0}}+N_{g} \frac{1-\cos \theta}{f \sin \theta} \vartheta^{\hat{3}}\right] \\
T^{\alpha}=\frac{k_{2}}{3} \vartheta^{\alpha} \wedge\left[N_{e}\left(1-\frac{1}{r}\right) \vartheta^{\hat{0}}+N_{g} \frac{1-\cos \theta}{f \sin \theta} \vartheta^{\hat{3}}\right] .
\end{gathered}
$$

The solutions presented in the section correspond to gravito-electric, gravito-magnetic, electric and magnetic charged monoples. 


\section{MONOPOLE SOLUTION IN SCHWARZSCHILD-LIKE COORDINATES}

As it is well known, the Schwarzschild coordinates are not always the more suitable coordinates for finding exact solutions. Nevertheless, we proceed in this section to write the monopole solutions of Sec. IV in Schwarzschild coordinates in order to compare them with the Reissner-Nordström solution of Sec. VII.

Therefore, let us rewrite the solution (4.2) in Schwarzschild coordinates $(\tau, \rho, \theta, \phi)$. In Eq. (3.3), we consider the explicit form of $\vartheta^{\hat{2}}$ and $\vartheta^{\hat{3}}$. Accordingly, we have to require [27]

$$
\rho=f r=[1 \pm q u(r)] r=(1 \pm q) r \mp q, \quad d r=\frac{d \rho}{1 \pm q} ; \quad \tau=\frac{t}{1 \pm q} .
$$

Thus

$$
f=\frac{1 \pm q}{g} ; \quad u(\rho)=1-\frac{1}{(\rho \pm q) /(1 \mp q)}=1-\frac{1 \mp q}{\rho \pm q}=\frac{\rho-1}{\rho \pm q}=\frac{1-1 / \rho}{g},
$$

where the function $g(\rho)$ reads

$$
g(\rho)=1 \pm \frac{q}{\rho}
$$

Therefore, in Schwarschild coordinates, the coframe turns out to be

$$
\vartheta^{\hat{0}}=g(\rho) d \tau, \quad \vartheta^{\hat{1}}=\frac{1}{g(\rho)} d \rho, \quad \vartheta^{\hat{2}}=\rho d \theta, \quad \vartheta^{\hat{3}}=\rho \sin \theta d \phi .
$$

It is orthonormal and the metric reads

$$
d s^{2}=o_{\alpha \beta} \vartheta^{\alpha} \otimes \vartheta^{\beta}=-g^{2} d \tau^{2}+\frac{1}{g^{2}} d \rho^{2}+\rho^{2}\left(d \theta^{2}+\sin ^{2} \theta d \phi^{2}\right)
$$

The electromagnetic potential (3.9) for this solution can be written as

$$
A=e_{o} \frac{1-1 / \rho}{g} \vartheta^{\hat{0}}+g_{o} \frac{1-\cos \theta}{\rho \sin \theta} \vartheta^{\hat{3}} .
$$

The relation

$$
q^{2}=\kappa\left(e_{o}^{2}+g_{o}^{2}+z_{4} k_{0}^{2} \frac{N_{e}^{2}+N_{g}^{2}}{2 a_{0}}\right)
$$

remains the same.

The nonmetricity and the torsion read as follows:

$$
\begin{gathered}
\left.Q^{\alpha \beta}=\left[k_{0} o^{\alpha \beta}+\frac{4}{9} k_{1}\left(\vartheta^{(\alpha} e^{\beta)}\right\rfloor-\frac{1}{4} o^{\alpha \beta}\right)\right]\left[N_{e} \frac{1-1 / \rho}{g} \vartheta^{\hat{0}}+N_{g} \frac{1-\cos \theta}{\rho \sin \theta} \vartheta^{\hat{3}}\right], \\
T^{\alpha}=\frac{k_{2}}{3} \vartheta^{\alpha} \wedge\left[N_{e} \frac{1-1 / \rho}{g} \vartheta^{\hat{0}}+N_{g} \frac{1-\cos \theta}{\rho \sin \theta} \vartheta^{\hat{3}}\right] .
\end{gathered}
$$

Now we are in position to present the generalized Reissner-Nordström solution in MAG and to compare it with our monopole solutions. 


\section{GENERALIZED REISSNER-NORDSTRÖM SOLUTION IN MAG}

In order to obtain the generalized Reissner-Nordström solution in MAG we choose a coframe of the Schwarzschild type 32

$$
\vartheta^{0}=f(r) d t, \quad \vartheta^{1}=\frac{1}{f(r)} d r, \quad \vartheta^{2}=r d \theta, \quad \vartheta^{3}=r \sin \theta d \phi
$$

which leads to the specific spherically symmetric metric

$$
d s^{2}=-f^{2} d t^{2}+\frac{1}{f^{2}} d r^{2}+r^{2}\left(d \theta^{2}+\sin ^{2} \theta d \phi^{2}\right)
$$

where the function $f(r)$ is unknown. The ansatz for the triplet of one-forms is now given by

$$
Q=k_{0}\left[N_{e} u(r, \theta) \vartheta^{\hat{0}}+N_{g} \frac{w(r, \theta)}{r \sin \theta} \vartheta^{\hat{3}}\right]=\frac{k_{0}}{k_{1}} \Lambda=\frac{k_{0}}{k_{2}} T .
$$

The ansatz for the electromagnetic potential turns out to be 30,31

$$
A=e_{o} u(r, \theta) \vartheta^{\hat{0}}+g_{o} \frac{w(r, \theta)}{r \sin \theta} \vartheta^{\hat{3}}
$$

where $e_{o}$ is the electric charge and $g_{o}$ the magnetic charge respectively.

For $\lambda \neq 0$ we obtain the following solution for the structure functions

$$
\begin{aligned}
f(r) & :=\sqrt{1-\frac{2 \mathrm{M}}{\mathrm{r}}+\kappa \frac{\mathrm{e}_{\mathrm{o}}^{2}+\mathrm{g}_{\mathrm{o}}^{2}+\left(\mathrm{z}_{4} / 2 \mathrm{a}_{0}\right) \mathrm{k}_{0}^{2}\left(\mathrm{~N}_{\mathrm{e}}^{2}+\mathrm{N}_{\mathrm{g}}^{2}\right)}{\mathrm{r}^{2}}-\frac{\lambda}{3 \mathrm{a}_{0}} \mathrm{r}^{2},} \\
u(r) & :=1 / f r \\
w(\theta) & :=(1-\cos \theta) .
\end{aligned}
$$

Thus, the electromagnetic potential has the following form

$$
\begin{aligned}
A & =e_{o} \frac{1}{f r} \vartheta^{\hat{0}}+g_{o} \frac{1-\cos \theta}{r \sin \theta} \vartheta^{\hat{3}} \\
& =e_{o} \frac{1}{r} d t+g_{o}(1-\cos \theta) d \phi .
\end{aligned}
$$

Moreover, the nonmetricity and the torsion can therefore be expressed as

$$
\begin{gathered}
\left.Q^{\alpha \beta}=\left[k_{0} o^{\alpha \beta}+\frac{4}{9} k_{1}\left(\vartheta^{(\alpha} e^{\beta)}\right\rfloor-\frac{1}{4} o^{\alpha \beta}\right)\right]\left[N_{e} \frac{1}{f r} \vartheta^{\hat{0}}+N_{g} \frac{1-\cos \theta}{r \sin \theta} \vartheta^{\hat{3}}\right] \\
T^{\alpha}=\frac{k_{2}}{3} \vartheta^{\alpha} \wedge\left[N_{e} \frac{1}{f r} \vartheta^{\hat{0}}+N_{g} \frac{1-\cos \theta}{r \sin \theta} \vartheta^{\hat{3}}\right] .
\end{gathered}
$$

Let us summarize the properties of the MAG-Maxwell solution presented here. The function $f$, which fixes the orthonormal coframe (6.1), has six contributions, see (6.5). The terms 
containing the mass parameter $M$, the cosmological constant $\lambda$, and the electric charge $e_{o}$ correspond exactly to the general relativistic Reissner-Nordström solution with cosmological constant. The term with the gravito-electric dilation charge $N_{e}$ has a similar structure as the previous term with the electric charge $e_{o}$. Additionally, we have in (6.5) a magnetic charge $g_{o}$ term and a gravito-magnetic charge $N_{g}$ term. Therefore, our solution is a generalizad Reissner-Nordström solution. It is clear that all relevant geometric objects, i.e. coframe, connection, torsion, curvature and nonmetricity feel via the function $f$ the presence of the electromagnetic field. However, otherwise the Maxwell field is disconnected from nonmetricity and torsion.

It is interesting to note that if we set $g_{o}=0$ and $N_{g}=0$ in the generalized ReissnerNordström solution presented above, see Eqs. Eqs. (6.5)-(6.10), the solution of Puntigam et al. 20] is obtained. Moreover, by setting $N_{g}=0, A=0$ the solution of Obukov et al. [18] is recovered. Besides, for $M=e_{o}$, i.e. mass equal to charge, and cosmological constant $\lambda=0$, it reduces to the monopole solution Eqs. (5.3), and (5.6)-(5.9). Additionally, by setting $N_{g}$ and $A=0$ in the charged monopole solution one recovers the vacuum gravito-electric charged monopole solution of Macías et al. [21], and the first term of the multipole solutions of Socorro et al. [22].

\section{FURTHER MULTIPOLE SOLUTIONS IN MAG}

Further solutions of the same type and in the framework of the same monopole configuration of Sec. III can be obtained by taking

$$
f=1 \pm q u(r, \theta), \quad u(r, \theta)=\frac{\cos \theta}{r^{2}}, \quad w(r, \theta)=\frac{\sin ^{2} \theta}{r},
$$

with an electromagnetic potential $A$ given by

$$
A=e_{o} \frac{\cos \theta}{r^{2}} \vartheta^{\hat{0}}+g_{o} \frac{\sin \theta}{r^{2} f} \vartheta^{\hat{3}} .
$$

The nonmetricity and the torsion can now be written as

$$
\begin{gathered}
\left.Q^{\alpha \beta}=\frac{1}{r}\left[k_{0} o^{\alpha \beta}+\frac{4}{9} k_{1}\left(\vartheta^{(\alpha} e^{\beta)}\right\rfloor-\frac{1}{4} o^{\alpha \beta}\right)\right]\left[N_{e} \frac{\cos \theta}{r} \vartheta^{\hat{0}}+N_{g} \frac{\sin \theta}{r f} \vartheta^{\hat{3}}\right], \\
T^{\alpha}=\frac{k_{2}}{3 r} \vartheta^{\alpha} \wedge\left[N_{e} \frac{\cos \theta}{r} \vartheta^{\hat{0}}+N_{g} \frac{\sin \theta}{r f} \vartheta^{\hat{3}}\right] .
\end{gathered}
$$

The relation

$$
q^{2}=\kappa\left(e_{o}^{2}+g_{o}^{2}+z_{4} k_{0}^{2} \frac{N_{e}^{2}+N_{g}^{2}}{2 a_{0}}\right),
$$

for $z_{4}$ still stands. These solutions are also endowed with gravito-electric $N_{e}$ and gravitomagnetic $N_{g}$ charges as well as with electric $e_{o}$ and magnetic $g_{o}$ charges.

Although the solutions presented in this section are not particular cases of the avobe presented generalized Reissner-Nordström solution, they are also interesting solutions which contribute to enhance our physical insight in the MAG theories.

All these solutions were checked with Reduce [33] with its Excalc package [34] for treating exterior differential forms [35] and the Reduce-based GRG computer algebra system [36, 37]. 


\section{CHARGE ASSIGNMEMT OF THE SOLUTIONS}

With propagating nonmetricity $Q_{\alpha \beta}$ two types of charge are expected to arise: One dilation charge related by the Noether procedure to the trace $Q:=Q_{\gamma}^{\gamma} / 4$ of the nonmetricity, called the Weyl covector $Q=Q_{i} d x^{i}$. It is the connection associated with gauging $R^{+}$instead of $U(1)$ in the case of the Maxwell potential $A=A_{i} d x^{i}$. Nine types of shear charge are related to the remaining traceless piece $\mathscr{Q}_{\alpha \beta}$ of the nonmetricity. Under the local Lorentz group, the nonmetricity can be decomposed into four irreducible pieces ${ }^{(I)} Q_{\alpha \beta}$, with $I=1,2,3,4$. The Weyl covector is linked to ${ }^{(4)} Q_{\alpha \beta}=Q g_{\alpha \beta}$. Therefore we should find $4+4+1$ shear charges and 1 dilation charge.

Besides mass $M$, monopole charges $e_{o}$ and $g_{o}$, our solution carries dilation, shear, and spin charges, each of them of the covectorial type. We have the following assignments:

$$
\begin{aligned}
& M \longrightarrow \text { mass of Schwarzschild type }, \\
& e_{o} \longrightarrow \text { electric charge } \\
& g_{o} \longrightarrow \text { magnetic charge } \\
& N_{e} \longrightarrow \text { gravito-electric charge }, \\
& N_{g} \longrightarrow \text { gravito-magnetic charge }, \\
& k_{0} N_{e}, k_{0} N_{g} \longrightarrow \text { dilation ('Weyl') charges of type }{ }^{(4)} Q^{\alpha \beta}, \\
& k_{1} N_{e}, k_{1} N_{g} \longrightarrow \text { shear charges of type }{ }^{(3)} Q^{\alpha \beta} \\
& k_{2} N_{e}, k_{2} N_{g} \longrightarrow \text { spin charges of type }{ }^{(2)} T^{\alpha} .
\end{aligned}
$$

In principle, however, these "charge" assignments need to be justified by integrating locally conserved Noether currents in MAG, such as Eq. (5.7.4) of Ref. 113.

\section{DISCUSSION}

The constants $a_{1}, a_{3} ; b_{1}, b_{2}, c_{2}$ do not occur in our Reissner-Nordström solution (6.5)(6.10), nor in the constraint (4.6), so they are irrelevant and we can put them to zero, Then the Lagrangian (3.1) reduces to

$$
\begin{aligned}
L & =V_{M A G}+V_{\text {Max }} \\
& =\frac{1}{2 \kappa}\left[-a_{0} R^{\alpha \beta} \wedge \eta_{\alpha \beta}-2 \lambda \eta+a_{2} T^{\alpha} \wedge^{*(2)} T_{\alpha}\right. \\
& +2\left(c_{3}{ }^{(3)} Q_{\alpha \beta}+c_{4}{ }^{(4)} Q_{\alpha \beta}\right) \wedge \vartheta^{\alpha} \wedge^{*} T^{\beta} \\
& \left.+Q_{\alpha \beta} \wedge^{*}\left(b_{3}{ }^{(3)} Q^{\alpha \beta}+b_{4}{ }^{(4)} Q^{\alpha \beta}\right)\right] \\
& -\frac{z_{4}}{2} R^{\alpha \beta} \wedge^{*(4)} Z_{\alpha \beta}-\frac{1}{2} F \wedge^{*} F,
\end{aligned}
$$

where $b_{4}$ is determined by (4.6). Consequently our solutions $(6.5)-(6.10)$, with $(4.3)-(4.7)$, solve also the field equations belonging to (9.1). However, for the monopole and multipole

solutions (4.2)-(4.10) and (7.1)-(7.5), respectively, we set additionally $\lambda=0$. It is interesting to note that, in (9.1) nonmetricity and torsion enter explicitly only with those pieces which are admitted according to (8.6), (8.7), (8.8). 
In view of 38,29, it is clear that one may start with any solution of the EinsteinMaxwell equations, then one replaces, after imposing a suitable constraint on the coupling constants, the electric and/or magnetic charge by strong gravito charges therby arriving at the post-Riemannian triplet. Now, one chooses an electromagnetic potential one-form $A$ proportional to the triplet one-form. Thus the structure of the energy-momentum of the triplet one-form and of the one-form $A$ is the same one. Moreover, both currents differ only by a constant. Explicit solutions convey a better undestanding of the structures involved.

In order to obtain solutions which excite more general post-Riemannian structures, it is desirable to go beyond the triplet ansatz (3.8), which requires a generalization of the Lagrangian (9.1).

The theories of modern physics generally involve a mathematical model, defined by a certain set of differential equations, and supplemented by a set of rules for translating the

mathematical results into meaninful statements about the physical world. In the case of gravity theories, because they deal with the most universal of the physical interactions, one has an additional class of problems concerning the influence of the gravitational field on other fields and matter. These are often studied by working within a fixed gravitational field, usually an exact solution [39]. In this context our generalized Reissner-Nordström solution and the monopole solutions contained on it contribute to enhance our understanding of some of these questions in the framework of MAG theories, in particular the ones concerned with the electrovacuum sector of them [11,31.

\section{ACKNOWLEDGMENTS}

We thank Friedrich W. Hehl for useful discussions and literature hints. This research was supported by CONACYT Grants 28339E and 3898P-E9608, and by the joint GermanMexican project DLR-Conacyt E130-2924 and MXI 009/98 INF . 


\section{REFERENCES}

[1] E.S. Fradkin and A.A. Tseytlin, Phys. Lett. B158 (1985) 316.

[2] C.G. Callan, D. Friedan, E.J. Martinec, and M.J. Perry, Nucl. Phys. 262 (1985) 593.

[3] D. Gross, Phys. Rev. Lett. 60 (1988) 1229.

[4] D. Gross and P.F. Mende, Nucl. Phys. B303 (1988) 407.

[5] D. Gross and P.F. Mende, Phys. Lett. B197 (1987) 129.

[6] A. Guth, Phys. Rev. D23 (1981) 347.

[7] A. Guth, Proc. Natl. Acad. Sci., USA 90 (1983) 4871.

[8] A. Linde, Phys. Lett. B108 (1982) 389.

[9] A. Linde, Phys. Lett. B249 (1990) 18.

[10] D. La and P.J. Steinhardt, Phys. Rev. Lett. 62 (1989) 376.

[11] Y. Ne'eman and F.W. Hehl, Class. Quantum Grav. 14 (1997) A251.

[12] F.W. Hehl, P. von der Heyde, G.D. Kerlick and J.M. Nester, Rev. Mod. Phys. 48 (1976) 393; F.W. Hehl, Found Phys. 15 (1985) 451.

[13] F.W. Hehl, J.D. McCrea, E.W. Mielke, and Y. Ne'eman, Phys. Rep. 258 (1995) 1.

[14] R. Tresguerres: Weyl-Cartan model for cosmology before mass generation, in Proceedings of the Relativity Meeting 1993, Relativity in General, Salas, Asturias, Spain. Sept. 7-10/1993. J. Díaz Alonso and M. Lorente Páramo, eds. (Ed. Frontiers, Gif-sur-Yvette 1994) pp. 407-413.

[15] R. Tresguerres, Z. Phys. C65 (1995) 347.

[16] R. Tresguerres, Phys. Lett. A200 (1995) 405.

[17] R.W. Tucker and C. Wang, Class. Quantum Grav. 12 (1995) 2587.

[18] Yu.N. Obukhov, E.J. Vlachynsky, W. Esser, R. Tresguerres, and F.W. Hehl, Phys. Lett. A220 (1996) 1.

[19] E.J. Vlachynsky, R. Tresguerres, Yu.N. Obukhov, and F.W. Hehl, Class. Quantum Grav. 13 (1996) 3253.

[20] R.A. Puntigam, C. Lämmerzahl, F.W. Hehl, Class. Quantum Grav. 14 (1997) 1347.

[21] A. Macías, E.W. Mielke and J. Socorro, Class. Quantum Grav. 15 (1998) 445.

[22] J. Socorro, C. Lämmerzahl, A. Macías, and E.W. Mielke, Phys. Lett. A244 (1998) 317.

[23] P. Baekler, E.W. Mielke, R. Hecht, and F.W. Hehl, Nucl. Phys. B288 (1987) 800.

[24] T. Dereli and R.W. Tucker, Class. Quantum Grav. 12 (1995) L31.

[25] A. García, F.W. Hehl, C. Lämmerzahl, A. Macías, and J.Socorro, Class. Quantum Grav. 15 (1998) 1793.

[26] A. García, C. Lämmerzahl, A. Macías, E.W. Mielke, and J. Socorro, Phys. Rev. D57 (1998) 3457.

[27] F. W. Hehl and A. Macías: Metric-affine gauge theory of gravity II. Exact solutions. (1999). Los Alamos preprint gr-qc/9902076. To appear in I. J. Mod. Phys. D.

[28] T. Dereli, M. Önder, J. Schray, R.W. Tucker, and C. Wang, Class. Quantum Grav. 13 (1996) L103.

[29] Yu.N. Obukhov, E.J. Vlachynsky, W. Esser, and F.W. Hehl, Phys. Rev. D56 (1997) 7769 .

[30] F.W. Hehl and J. Socorro, Acta Phys. Pol. 29 (1998) 1113.

[31] A. García, A. Macías, and J. Socorro, Class. Quantum Grav. 16 (1999) 93.

[32] A. García and A. Macías: "Black holes as solutions of the Einstein-Maxwell equations of Petrov type D", in: Black Holes: Theory and Observations. F.W. Hehl, C. Kiefer 
and R.J.K. Metzler, eds. Lecture Notes in Physics. Vol. 514 (Springer Verlag 1998) pp. 203-224.

[33] A.C. Hearn, REDUCE User's Manual. Version 3.6. Rand publication CP78 (Rev. 7/95) (RAND, Santa Monica, CA 90407-2138, USA, 1995).

[34] E. Schrüfer, F.W. Hehl, and J.D. McCrea, Gen. Relat. Grav. 19 (1987) 197.

[35] D. Stauffer, F.W. Hehl, N. Ito, V. Winkelmann, and J.G. Zabolitzky: Computer Simulation and Computer Algebra - Lectures for Beginners. 3rd ed. (Springer, Berlin, 1993).

[36] V.V. Zhytnikov, GRG. Computer Algebra System for Differential Geometry, Gravity and Field Theory. Version 3.1 (Moscow, 1991) 108 pages.

[37] J. Socorro, A. Macías, and F.W. Hehl, Computer Physics Communications 115 (1998) 264.

[38] R. Tucker, C. Wang: Nonriemannian gravitational interactions. Talk given at Mathematical Aspects of Theories of Gravitation, Warsaw, Poland (1996). Banach Centre Publications Vol. 41 (Institute of Mathematics, Polish Academy of Sciences, Warsaw 1997). Los Alamos E-Print Archive: gr-qc/9608055.

[39] D. Kramer, H. Stephani, M. MacCallum, and E. Herlt: Exact Solutions of the Einstein Field Equations. (Deutsche Verlag der Wiessenschaften, Berlin 1980). 